\title{
REVIEW OF GASTRIC TORSION IN EIGHT GUINEA PIGS (CAVIA PORCELLUS)
}

\author{
Anna Linda NóGRÁDI ${ }^{1 *}$, Iain COPE ${ }^{2}$, Márton BALOGH ${ }^{3}$ and János GÁL ${ }^{1}$ \\ ${ }^{1}$ Department and Clinic of Exotic Animal and Wildlife Medicine, University of Veterinary \\ Medicine, István u. 2, H-1078 Budapest, Hungary; ${ }^{2}$ Vets4Pets Newmarket, inside Pets at \\ Home, Studland Retail Park, Fordham Road, Newmarket, Suffolk, UK; ${ }^{3}$ Department and \\ Clinic of Internal Medicine, University of Veterinary Medicine, Budapest, Hungary
}

(Received 8 April 2017; accepted 6 November 2017)

The authors present eight cases of gastric dilatation and volvulus (GDV) in guinea pigs from the Department and Clinic of Exotic Animal and Wildlife Medicine, University of Veterinary Medicine, Budapest, Hungary between 2012 and 2016. Seven animals were operated on and two survived. Gastric torsion has been noted in many mammalian species. Gastric volvulus has a high morbidity and high mortality rate with a guarded to poor prognosis in all of these species. How GDV develops is still not widely understood. Postmortem examinations, in both our cases and previously reported cases, have failed to reveal the exact causes of the gastric torsions. The aetiology of gastric torsion in guinea pigs is probably multifactorial. Feeding fewer meals per day, eating rapidly, decreased food particle size, exercise, stress after a meal, competition, age, and an aggressive or fearful temperament, are all likely and potential risk factors for GDV development in a similar fashion to dogs. Sex, breeding, dental diseases, anatomical abnormalities, pain and pregnancy may also be contributing factors. treatment

Key words: Guinea pig, gastric dilatation, volvulus, GDV, diagnosis,

Gastric torsion has been described in many species (Richardson, 2000; Hedlund and Fossum, 2007; Cornell, 2012). Gastric dilatation and volvulus (GDV) also occurs in guinea pigs (Richardson, 2000; Hawkins, 2012).

The cause of GDV is generally unknown. However, size, age, history of GDV in a first-degree relative, feeding fewer meals per day, eating rapidly, decreased food particle size, an aggressive or fearful nature, and exercise or stress after meals, have all been mentioned as risk factors to gastric torsion in dogs (Richardson, 2000; Hedlund and Fossum, 2007).

GDV is a life-threatening condition with a high morbidity and high mortality rate. The stomach rotates around its axis, and thus a functional obstruction occurs. Fluid and gas gets trapped in the lumen and bacterial fermentation in the

\footnotetext{
*Corresponding author; E-mail: anna.nogradi@gmail.com; Phone: 0036 (1) 478-4100
} 
stomach causes further gastric distension leading to increased abdominal pressure. This decreases venous flow in the caudal vena cava and in the portal vein, causing portal hypertension, systemic hypotension, venous stasis and functional disorders of the heart. This in turn can lead to cardiogenic shock. Venous stasis itself causes mucosal death in the stomach and bacterial dislocation. Pressure of the distended stomach on the diaphragm makes inspiration difficult (Hedlund and Fossum, 2007; Cornell, 2012).

Bloating and hypomotility of the intestines can lead to gastric torsion in guinea pigs and can be caused by various factors. The real cause is usually unknown, but bezoars, poor-quality feed, bacterial infections, various drugs, pain, and dental disease can all lead to anorexia and bloating (Michelle, 2009).

Clinical signs of gastric torsion in guinea pigs include abdominal distension and tympany, abdominal pain, no intestinal sounds during auscultation, short sharp shallow breaths, and cardiovascular signs. The animals are anorexic, dull, weak and depressed with a poor hydration status and decreased to no faecal output. Sudden death can occur (Richardson, 2000).

Orthogonal radiographic imaging can be helpful in the differential diagnosis of gastric torsion in guinea pigs. The gas-distended stomach can fill over 50\% of the abdomen and may be seen on the right side of the image (Dudley and Boivin, 2011; DeCubellis and Graham, 2013; Nógrádi et al., 2015). Confirmation of a gastric torsion can be made through laparotomy or postmortem examination.

In the case of an extremely bloated stomach, a stomach tube or trochar should be used to deflate the stomach. There is a high risk of peritonitis when performing trocharisation in guinea pigs. Prognosis is guarded or poor in case of bloating and hypomotility in guinea pigs (Michelle, 2009; Hawkins, 2012). Gastric volvulus itself is a life-threatening condition that needs to be treated immediately. Surgery is the treatment of choice, but the prognosis is poor even with this (Hawkins, 2012). To the best of our knowledge, in the current literature all the guinea pigs affected by gastric volvulus died, were euthanised, or were diagnosed during postmortem reviews.

\section{Literature review on cases of gastric dilatation and volvulus in guinea pigs}

There is one report of two gastric torsions in a breeding colony of 36 animals within a time span of two years; both cases were diagnosed post mortem. Both animals were female and had given birth already. One animal was pregnant again at the time of torsion and death (Dudley and Boivin, 2011).

Another report describes six breeding animals with gastric volvulus in a colony of an average of 253 animals within a time span of 18 months. Unfortunately the sex and the breeding status are not specified in that article. Clinical signs included tachycardia, dyspnoea, cyanosis and bloating (Lee et al., 1977).

A three-year-old female animal was diagnosed with GDV with the help of diagnostic imaging in another case. The veterinarians recommended immediate 
surgery, but the owner declined explorative laparotomy and asked for euthanasia. Unfortunately, even the postmortem examination could not reveal the cause of the GDV in this animal (Mitchell et al., 2010).

One publication mentions a female guinea pig that had both a gastric and a uterine torsion diagnosed at the time of death (Kunstyr, 1981).

A report of a non-fasted gravid female anaesthetised for jugular venous catheter placement also describes the development of gastric volvulus in the acute postoperative period. The animal was anaesthetised with ketamine and xylazine. The inhibitory effect pf xylazine on gastrointestinal motility, the nonfasted ingesta-filled stomach, and the positional changes during the procedure could have contributed to the development of the condition (Keith et al., 1992).

One case of dilatation and four cases of dilatation and volvulus occurred in a colony of 10 male and 19 female breeding animals within a one-year time frame. All animals were deficient in complement 4 . The predisposition of this strain is considered in the publication. One of the affected animals was a male guinea pig (Gialamas et al., 1985).

With the increasing number of GDV cases in guinea pigs seen by the authors and the scant literature available on the topic, most of which involves guinea pigs in research, the goal of the study was to compare our findings with those reported in the literature, get an insight into the aetiology of this disease and try to find an answer to the question why the number of guinea pigs affected by GDV has increased in recent years.

\section{Materials and methods}

\section{Case reports}

The authors diagnosed eight cases of GDV at the Department and Clinic of Exotic Animal and Wildlife Medicine, University of Veterinary Medicine, Budapest, Hungary between 2012 and 2016 with two animals surviving the torsions (see Table 1).

\section{Diagnostic imaging}

Radiographic examinations with orthogonal projections were performed in every case. In cases where the gastric torsion was $180^{\circ}$, the displacement of the stomach to the right side of the abdomen could easily be seen, and gastric torsion could be suspected immediately. In the case of partial torsions, radiographic images were not so easy to evaluate (Figs 1 and 2). Barium sulphate contrast medium was used in these cases (Fig. 3). 


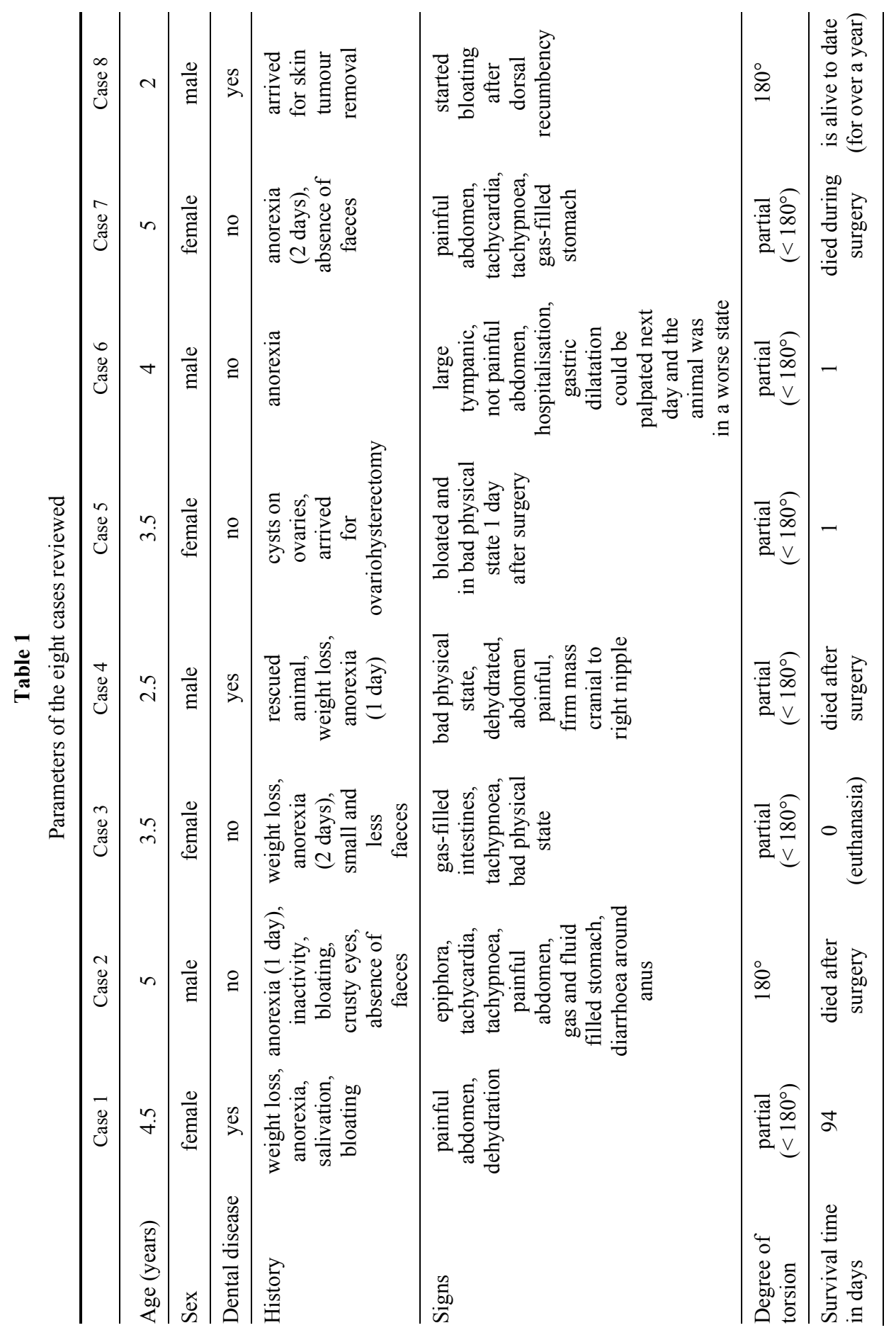




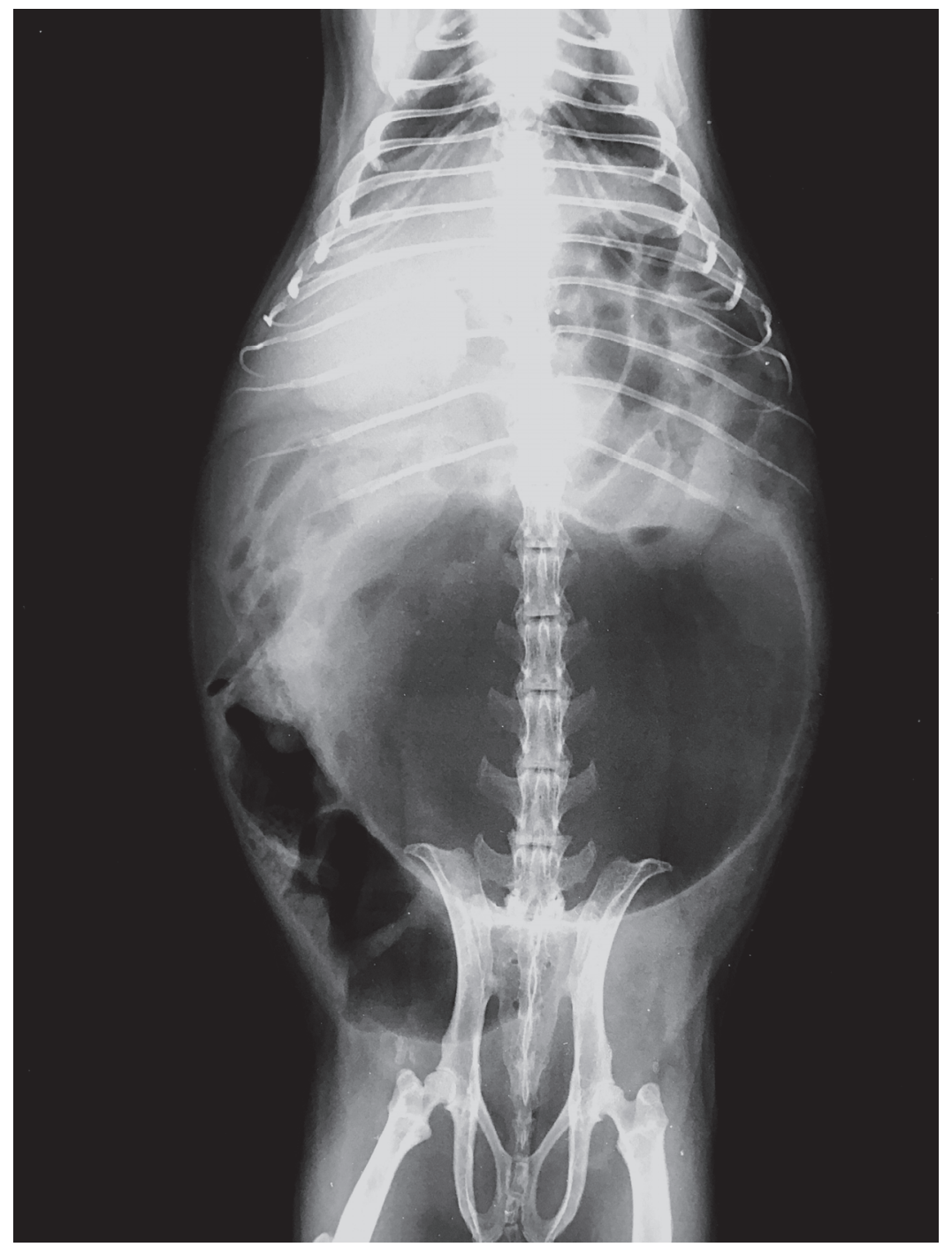

Fig. 1. The laterolateral radiograph of Case 3 shows the dilated and gas-filled stomach. The small intestines can be seen in front of the stomach

\section{Surgery and postoperative treatment}

All animals treated surgically were premedicated using ketamine $(3 \mathrm{mg} / \mathrm{kg})$, dexmedetomidine $(0.1 \mathrm{mg} / \mathrm{kg})$ and butorphanol $(0.3 \mathrm{mg} / \mathrm{kg})$ injected intramuscularly [IM], before being oxygenated. An intravenous catheter was placed in the cephalic vein when possible, allowing $15 \mathrm{ml} 0.9 \%$ sodium chloride to be given by infusion. Infusion fluid containing amino acids, vitamins and electrolytes (Duphalyte, $5 \mathrm{ml}$ ) was also given. 


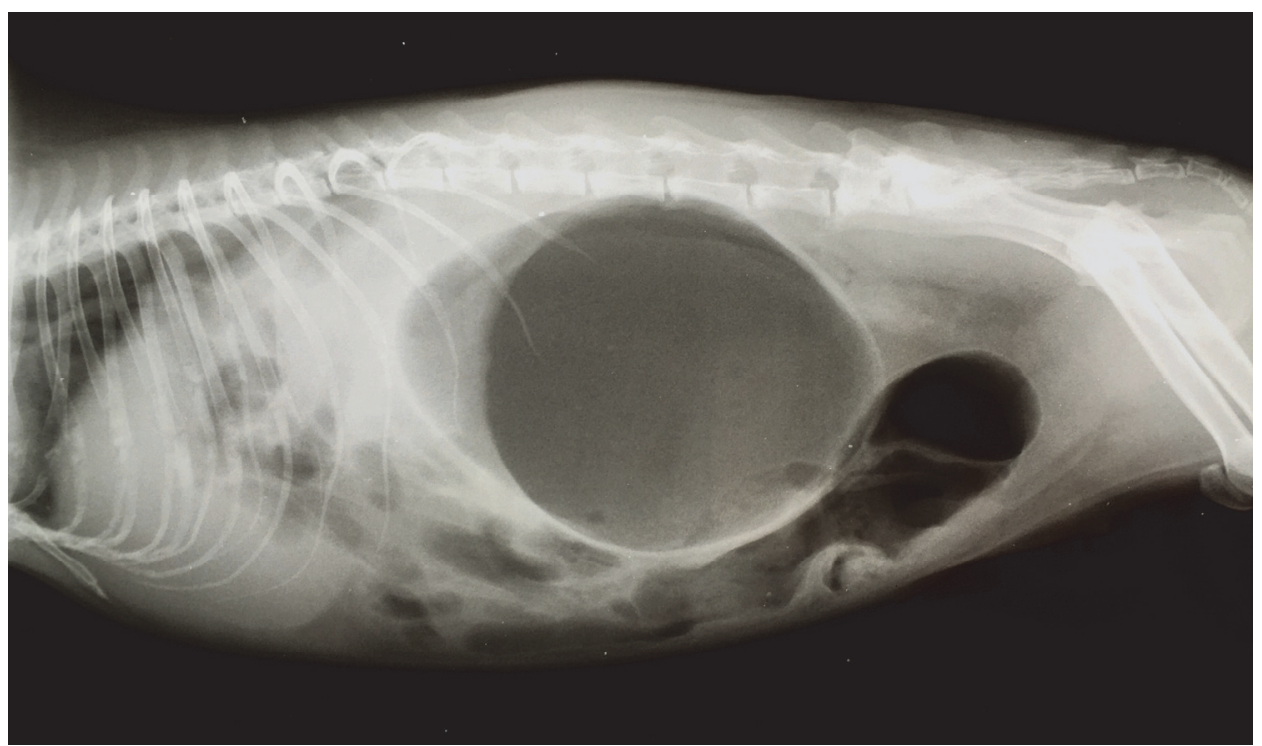

Fig. 2. Ventrodorsal radiograph of Case 3. The gas-filled stomach is displaced and the small intestines are cranial to the stomach

General anaesthesia was induced with $5 \mathrm{v} / \mathrm{v} \%$ isoflurane, before being maintained with $2.5 \mathrm{v} / \mathrm{v} \%$ isoflurane using $100 \%$ oxygen at $1.5 \mathrm{~L}$ flow via facemask. Endoscopy for immediate intubation was at hand at all times in case it was needed. Hair was clipped from the surgical site and the animal was placed in dorsal recumbency on the heated surgical table. The surgical site was scrubbed with Bradonett disinfectant liquid, treated with Bradoderm Soft hand and skin disinfectant, and then isolated using a sterile surgical foil drape.

All explorative laparotomies were performed through a medial incision (Figs 4 and 5). The stomach was located and assessed and an incision between the greater and lesser curvature on the ventral aspect of the stomach was made. The stomach content was then removed via gastrotomy. After emptying and flushing, the stomach was closed in two layers using PDS 4-0 USP (1.5 metric) in a two-layer inverting seromuscular pattern. The small intestines were replaced in their anatomical position within the abdomen and the stomach was repositioned. Abdominal lavage ( $0.9 \%$ sodium chloride) was performed, after which the abdominal wound was closed using Vicryl Plus 3-0 USP (2 metric), in three layers using continuous patterns. We attempted gastropexy in the line of the linea alba in some cases, while in others we did not, while closing the wound. The cases that survived did not have gastropexy.

Postoperative management included injections of meloxicam $(0.2 \mathrm{mg} / \mathrm{kg}$ subcutaneously [SC]), metoclopramide ( $1 \mathrm{mg} / \mathrm{kg} \mathrm{SC})$, enrofloxacin $(10 \mathrm{mg} / \mathrm{kg} \mathrm{SC})$ and fluid infusion therapy. The animals stayed on a heating mat until normal body temperature was back to normal and maintained. 


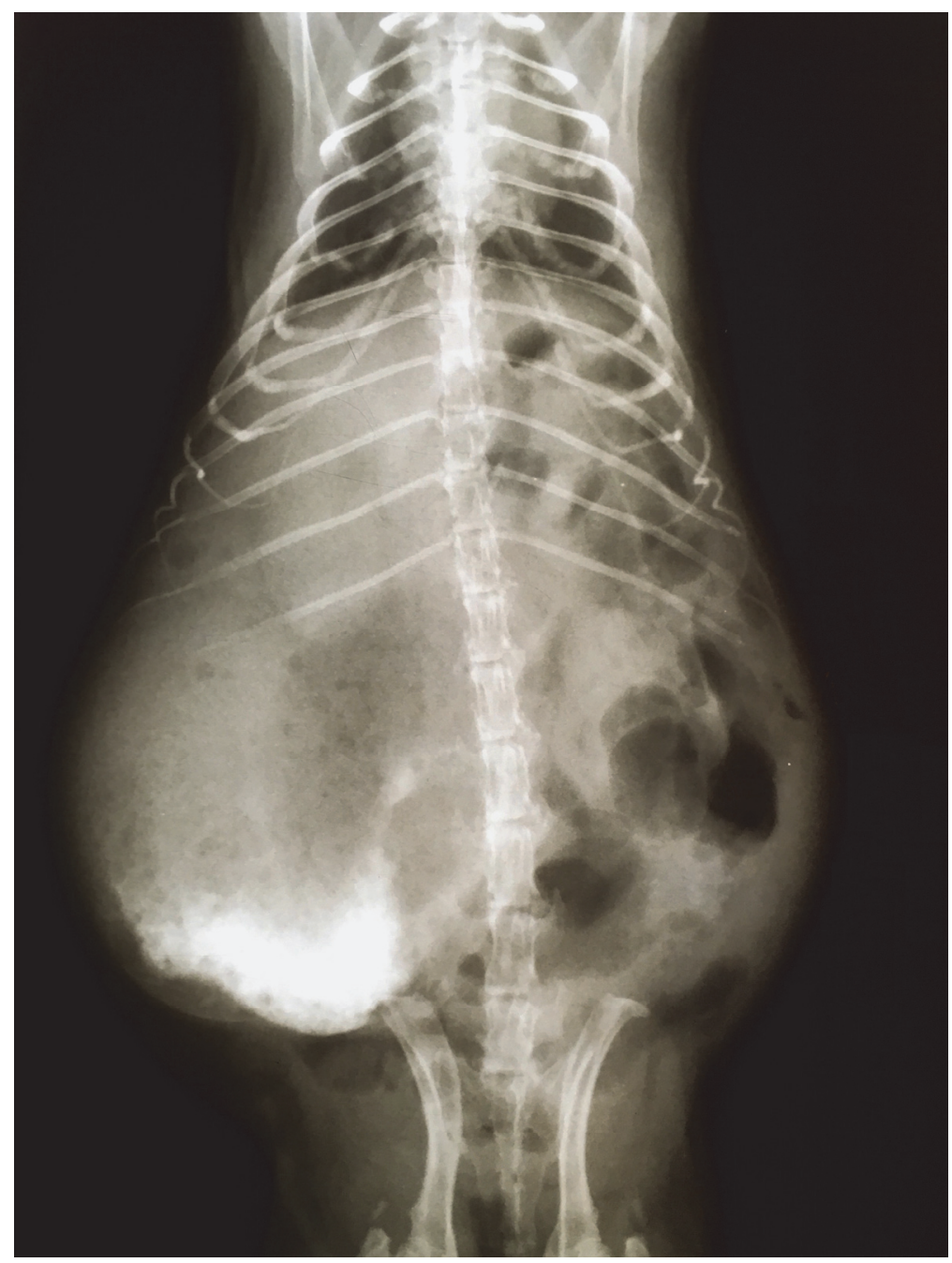

Fig. 3. Ventrodorsal radiograph of Case 6 after applying barium sulphate contrast medium. The stomach can be seen on the right side of the abdomen

\section{Pathology}

Case 3 was euthanised and sent for pathology. The abdomen was tense upon palpation. After opening the abdomen, a gas-filled stomach could be seen, that filled the epigastrium and mesogastrium (Fig. 6). Upon examining the abdominal organs, the spleen was enlarged, dark brownish red in colour and displaced to the right abdomen. The bile duct was permeable, and the liver was normally shaped and coloured, with the visceral surface pale brownish red. Upon opening the distended and displaced stomach a large amount of gas departed and about $8-10 \mathrm{ml}$ of red, slightly smelly content was left in the stomach. Suffusion- 
like bleeding could be seen from the serosal surface of the stomach. The mucous membrane was dark greyish red, and mildly broader than normal. There was a greyish-white ring at the cardiac and pyloric orifices. The content of the small and large intestines was normal. Abnormalities in other organs could not be seen.

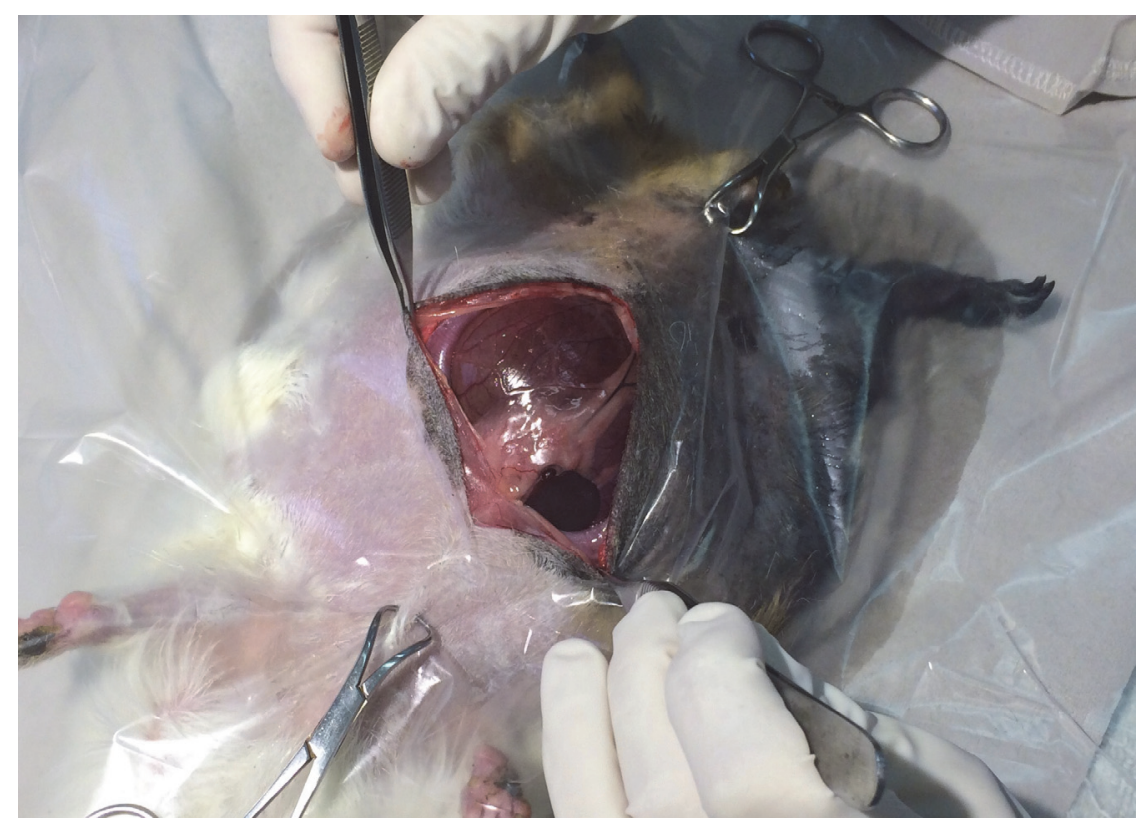

Fig. 4. Intraoperative photograph of a guinea pig with gastric torsion (Case 2). The dilated, poorly perfused stomach and the spleen can be seen on the right side of the abdomen

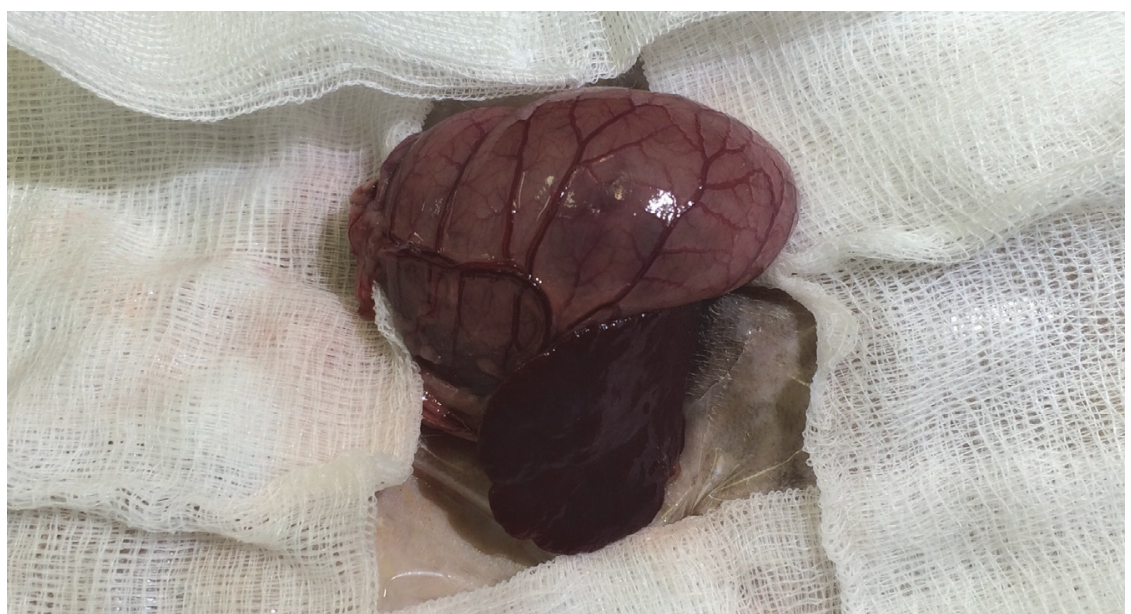

Fig. 5. Intraoperative photograph of a guinea pig with partial gastric torsion. The spleen is situated on the right side of the abdomen instead of its anatomical place because of the $90^{\circ}$ torsion. Blood supply to the stomach is also compromised 


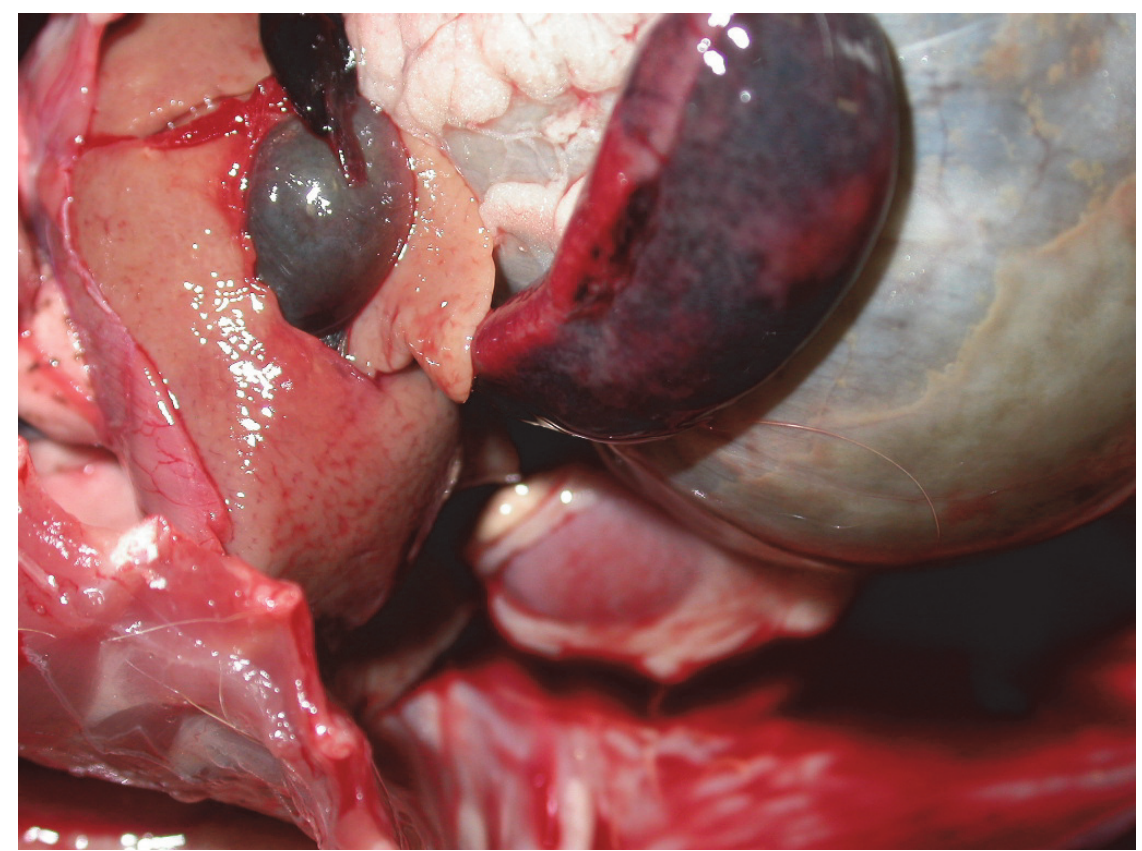

Fig. 6. Pathological findings of a guinea pig with gastric torsion (Case 3). The stomach and the spleen can be seen on the right side of the abdominal cavity

\section{Results}

Case 1 survived 94 days after diagnosis. Case 8 survived over one and a half years and is still healthy. All the other cases passed away between 0 and 1 day after diagnosis.

Case 1 was kept in hospital for 7 days after surgery. Treatment in the hospital included butorphanol $(0.3 \mathrm{mg} / \mathrm{kg} \mathrm{SC})$, meloxicam, drotaverine hydrochloride ( $2 \mathrm{mg} / \mathrm{kg} \mathrm{IM})$, metoclopramide, and enrofloxacin. Infusion therapy, vitamin B (NEOVIT B Complex injection, $0.2 \mathrm{ml} \mathrm{SC}$ ), and butaphosphan/cyanocobalamin (Catosal injection, $0.2 \mathrm{ml} \mathrm{SC}$ ) were also given daily. The animal was additionally syringe fed (TROVET Guinea Pig diluted with water, $15 \mathrm{ml}$, by mouth, 3-4 times per day) for the length of its hospitalisation. The patient was discharged from the hospital on the seventh day after surgery with a guarded long-term prognosis. Faeces was normal and the animal was feeding on its own. The animal was to be fed at home with the same amount as had been given in the hospital in case of ongoing anorexia. Medication for ongoing care included meloxicam, enrofloxacin and metoclopramide for five days; as well as simethicone (20 mg twice daily by mouth for 14 days), probiotics (Bio Lapis for Rabbits, 1 sachet per day by mouth), and silymarin (20 mg daily, by mouth). A strict diet was pre- 
scribed with ad libitum hay, a small amount of pellets for guinea pigs and an occasional small amount of vegetables cut into small pieces. The animal was examined 5 days, 3 weeks and 6 weeks after being discharged from the hospital, respectively. At these examinations the faeces was normal and the animal was feeding on its own, but it was not able to put on any extra weight. The stomach was normally placed and doughy, but palpably enlarged. The guinea pig died suddenly, 94 days after surgery. Unfortunately, the cause of death could not be determined, because the owner declined the postmortem examination.

Case 8 received the same treatment as Case 1, apart from drotaverine hydrochloride which was not used, and recovered without complications at home with the above-described treatment, but also receiving tramadol $(2.5 \mathrm{mg}$ by mouth, twice daily).

\section{Discussion}

Gastric dilatation and volvulus has been noted in some mammalian species. Gastric volvulus has a high morbidity and high mortality rate with a guarded to poor prognosis in all of these species. Most of the literature can be found on canines, swine and humans where gastric volvulus has been published more frequently than in other species (Dudley and Boivin, 2011). GDV is not common in guinea pigs, and is often diagnosed at postmortem examination (Dudley and Boivin, 2011).

How GDV develops is clearly still not widely understood. Postmortem examinations in both the previously reported cases and in our case failed to reveal the exact causes of the gastric torsions. Most of the reports of guinea pigs with gastric torsions involve female animals and many were either gravid or had previously bred. The authors, however, did not experience this sex-related predisposition in their presented cases, where in fact half of the gastric torsions presented were male animals and none of the female cases had a history of pregnancy at the time of or shortly before the gastric torsion.

When considering the possibility of a sex-related bias, it is worth noting that the uterus of a gravid dam can weigh as much as $50 \%$ of the animal's body weight (Ganaway and Allen, 1971). Gastric ligament lengthening associated with pregnancy could thus be a predisposing factor in this species (Dudley and Boivin, 2011). Ovarian cysts can also develop into very large space-occupying abdominal masses, which, just like pregnancy, rearrange the organs of the abdomen. This could also lead to gastric ligament lengthening. Case 1 was gravid when bought from the pet store years prior to the gastric torsion, and thus this may have lengthened the ligaments setting up a predisposition to future torsion.

GDV occurred in the acute postoperative period after ovariohysterectomy in Case 5 and during surgical preparation and dorsal recumbency in Case 8. The non-fasted gravid female reported in the literature, anaesthetised for jugular ve- 
nous catheter placement with ketamine and xylazine, also developed gastric volvulus in the acute postoperative period (Keith et al., 1992). The anaesthesia protocol of the authors also includes alpha-2 agonists (dexmedetomidine), but xylazine is not used because of its intense effect on the motility of the gastrointestinal tract. Stress of the hospitalisation and postoperative pain could have both contributed to the development of gastric volvulus in Case 5, despite the use of NSAIDS/opioids.

Gastric torsion in cats is often associated with trauma-related anatomic abnormalities (Formaggini et al., 2008). Anatomical abnormalities including abnormal lengthening of the gastric ligaments and congenital or acquired upper abdominal defects are the cause of gastric volvulus in humans (Askew, 1978). The cases presented by the authors were not exposed to trauma nor did postmortem examinations reveal any anatomical abnormalities. An ovarian cyst can, however, be seen as an anatomical abnormality that can also cause the lengthening of the gastric ligaments, and so this may be a predisposing factor as discussed previously.

Guinea pigs are predisposed to dental diseases. When a guinea pig with anorexia is presented to the clinic, dental disease must always be ruled out since gastric stasis can occur secondary to dental problems and the resulting anorexia (Dudley and Boivin, 2011). Three of the guinea pigs presented did have malocclusion and were treated regularly for tooth trimming.

Stress as a predisposing factor has been linked to GDV in dogs and swine. Stress can be caused by various factors including social isolation, high animal density, suppression by a dominant animal, and competition for food. Children can also be considered as a stress factor for guinea pigs, which are often fed after the children arrive home from school and then picked up and played with. Children also may rotate the animals while playing, and do not hold the animals in their normal anatomical position. This may also contribute to the development of gastric torsion. However, there were no children in the household in most of our presented cases and children did not rotate these animals after eating.

Cases 2-4 were all presented to the clinic within a single week. The weather was not typical of the season with rapidly changing temperatures from below freezing point to temperatures as high as $15^{\circ} \mathrm{C}$. This also could have caused stress in the animals and thus affected the development of dilation and torsion.

Feeding few meals per day, exercise and stress are all identified risk factors in dogs, which can lead to gastric volvulus (Hedlund and Fossum, 2007; Cornell, 2012). Overeating with dry food, followed by excessive drinking, was listed as a possible cause of gastric torsion in guinea pigs (Gialamas et al., 1985), although this has not been proved. Competition for food can lead to both rapid and excess ingestion of food items. All animals were fed with ad libitum hay and water, whilst pellets were given regularly in small amounts. Vegetables were given occasionally. It is possible that pellets that are a scarce resource can lead to competition within colonies, thus motivating the animals to eat rapidly. This 
competition could also be a stress factor. The experience of the authors is that guinea pigs consuming large amounts of dry pellets, also consume large amounts of water afterwards, probably due to the dryness of the feed. Pellets show extreme swelling when soaked in water, and thus may rapidly expand in the gastrointestinal tract if eaten and swallowed quickly, rather than being chewed slowly. This would rapidly expand and dry the stomach, as well as the mouth, thus likely explaining the need to drink more after eating these pellets. It is worth noting that commercially available guinea pig food has changed in the past few years. Seed and crushed mixes were often used to feed guinea pigs, but recently compressed pellets have become commercially accessible. These do have a decreased particle size which again may have an impact on gastrointestinal function, motility, and transit times when compared to hay and other long-fibre graze diets.

\section{Clinical significance}

The aetiology of gastric torsion in guinea pigs is probably multifactorial. Feeding fewer meals per day, eating rapidly, decreased food particle size, exercise, stress after a meal, competition, age, and an aggressive or fearful temperament, are all likely and potential risk factors for GDV development in a similar fashion to dogs. Sex, breeding, dental diseases, anatomical abnormalities, pain and pregnancy may also be factors, but further research is required with larger numbers of cases to clarify this.

\section{References}

Askew, A. R. (1978): Treatment of acute and chronic gastric volvulus. Ann. R. Coll. Surg. Engl. 60, 326-328.

Cornell, K. (2012): Stomach. In: Tobias, K. M. and Johnston, S. A. (eds) Veterinary Surgery: Small Animal. Saunders, Elsevier, St. Louis. pp. 1484-1512.

DeCubellis, J. and Graham, J. (2013): Gastrointestinal disease in guinea pigs and rabbits. Vet. Clin. North Am. Exot. Anim. Pract. 16, 421-435.

Dudley, E. S. and Boivin, G. P. (2011): Gastric volvulus in guinea pigs: comparison with other species. J. Am. Assoc. Lab. Anim. Sci. 50, 526-530.

Formaggini, L., Schmidt, K. and De Lorenzi, D. (2008): Gastric dilatation-volvulus associated with diaphragmatic hernia in 3 cats: clinical presentation, surgical treatment, and presumptive aetiology. J. Feline Med. Surg. 10, 198-201.

Ganaway, J. R. and Allen, A. M. (1971): Obesity predisposes to pregnancy toxemia (ketosis) of guinea pigs. Lab. Anim. Sci. 21, 40-44.

Gialamas, J., Höger, H. and Adamiker, D. (1985): Akute Magendilatation und Magendrehung beim Meerschweinchen [in German]. Zentralbl. Veterinarmed. Reihe A 32, 772-777.

Hawkins, M. G. (2012): Disease problems of guinea pigs. In: Quesenberry, K. E. and Carpenter, J. W. (eds) Ferrets, Rabbits, and Rodents: Clinical Medicine and Surgery. Saunders, Elsevier, St. Louis. pp. 295-310.

Hedlund, Ch. S. and Fossum, T. W. (2007): Surgery of the Stomach. In: Fossum, T. W. (ed) Small Animal Surgery. Third edition. Saunders, Elsevier, St. Louis. pp. 409-442.

Keith, J. C., Rowles, T. K., Warwick, K. E. and Yau, E. T. (1992): Acute gastric distention in guinea pigs. Lab. Anim. Sci. 4, 331-332. 
Kunstyr, I. (1981): Torsion of the uterus and the stomach in guinea pigs. Z Versuchstierkd. 23, 67-69.

Lee, K. J., Johnson, W. D. and Lang, C. M. (1977): Acute gastric dilatation associated with gastric volvulus in the guinea pig. Lab. Anim. Sci. 27, 685-686.

Michelle, L. W. (2009): Rodents: digestive system disorders. In: Keeble, E. and Meredith, A. (eds) BSAVA Manual of Rodents and Ferrets. BSAVA, Gloucester. pp. 123-141.

Mitchell, E. B., Hawkins, M. G., Gaffney, P. M. and MacLeod, A. G. (2010): Gastric dilatationvolvulus in a guinea pig (Cavia porcellus). J. Am. Anim. Hosp. Assoc. 46, 174-180.

Nógrádi, A. L., Csikó, Gy. and Gál, J. (2015): Gastric dilatation and volvulus in a guinea pig (Cavia porcellus): Case report [in Hungarian, with English abstract]. Magy Allatorvosok 137, 159-165.

Richardson, V. C. G. (2000): The digestive system. In: Richardson, V. C. G. (ed.) Diseases of Domestic Guinea Pigs. 2nd edition. Blackwell Publishing. pp. 50-61. 